\title{
The influence of dietary fatty acid composition on $N$-ethyl- $N$ - nitrosourea-induced mammary tumour incidence in the rat and on the composition of inositol- and ethanolamine-phospholipids of normal and tumour mammary tissue
}

\author{
BY CHRISTINEM. WILLIAMS AND KAREN MAUNDER \\ The Nutritional Metabolism Research Group, School of Biological Sciences, University of Surrey, \\ Guildford GU2 $5 X H$
}

(Received 10 February 1993 - Revised 19 April 1993-Accepted 21 June 1993)

\begin{abstract}
This study has investigated the influence of dietary fatty acid composition on mammary tumour incidence in $N$-ethyl- $N$-nitrosourea (ENU)-treated rats and has compared the susceptibility to dietary fatty acid modification of the membrane phospholipids phosphatidylinositol (PI) and phosphatidylethanolamine (PE) from normal and tumour tissue of rat mammary gland. The incidence of mammary tumours was significantly lower in fish oil- $(29 \%)$, compared with olive oil- $(75 \% ; P<0.04)$ but not maize oil$(63 \% ; P<0.1)$ fed animals. No differences in PI fatty acid composition were found in normal or tumour tissue between rats fed on maize oil, olive oil or fish oil in diets from weaning. When normal and tumour tissue PI fatty acids were compared, significantly higher amounts of stearic acid (18:0) were found in tumour than normal tissue in rats given olive oil $(P<0.05)$. A similar trend was found in animals fed on maize oil, although differences between normal and tumour tissue did not reach a level of statistical significance $(P<0.1)$. In mammary $P E$, maize oil-fed control animals had significantly higher levels of linoleic acid (18:2n-6) than either olive oil- or fish oil-fed animals $(P<0.05$, both cases) and levels of arachidonic acid were also higher in maize oil- compared with fish oil-fed animals $(P<0.05)$. In tumourbearing animals no differences in PE fatty acid composition were found between the three dietary groups. When normal and tumour tissue PE fatty acids were compared, significantly lower amounts of linoleic acid (18:2n-6; $P<0.01)$ and significantly greater amounts of arachidonic acid $(20: 4 n-6 ; P<0.05)$ were found in tumour than normal tissue of rats fed on maize oil. The present study shows that the fatty acid composition of PI from both normal and tumour tissue of the mammary gland is resistant to dietary fatty acid modification. The PE fraction is more susceptible to dietary modification and in this fraction there is evidence of increased conversion of linoleic acid to arachidonic acid in tumour compared with normal tissue. Lower tumour incidence rates in rats given fish oils may in part be due to alteration in prostanoid metabolism secondary to displacement of arachidonic acid by eicosapentaenoic acid, but $P E$ rather than PI would appear to be the most likely locus for diet-induced alteration in prostanoid synthesis in this tissue. Effects of dietary fatty acids other than on the balance of $n-6$ and $n-3$ fatty acids, and on prostanoid metabolism, should also be considered. The significance of increased stearic acid content of PI in tumours of olive oil-fed animals and the possible influence of dietary fatty acids on the capacity for stearic acid accumulation requires further study.
\end{abstract}

Fatty acids: Phospholipids: Mammary tumour: Rat

Several international cross-cultural studies have reported associations between dietary fat intake and mortality and morbidity from breast cancer (Lea, 1966; Carroll, 1974). However, recent case-control and cohort studies have produced conflicting findings; some have found no evidence of higher fat intakes in women with breast cancer whilst others have demonstrated a positive association with fat intake (Berrino \& Muti, 1989). The use of case-control studies as a means of investigating the relationship between diet and breast 
cancer has been questioned, particularly in populations with narrow ranges of fat intake (Prentice et al. 1988). Furthermore, such studies assume a linear relationship between fat intake and cancer risk, an assumption which may be invalid (McMichael \& Potter, 1985; Potter, 1987). For these reasons, further studies in animals are required to provide a better understanding of the biochemical mechanisms that underlie effects of dietary fat on this disease. Such studies may enable the design of more appropriate studies in human populations.

Recent animals studies suggest that at least some of the tumour-enhancing effects of high-fat diets are due to provision of excess energy (Kritchevsky et al. 1984; Welsch et al. 1990). However, the type of fat fed also appears to be important since $n-3$ fatty acids present in fish oils have been shown to inhibit the growth of transplanted tumours (Karmali et al. 1984) and to reduce the incidence rates of carcinogen-induced tumours (Jurkowski \& Cave, 1985). Protective effects of $n-3$ fatty acids have been attributed to reduced synthesis of series 2 prostaglandins resulting from the displacement of arachidonic acid (AA), from membrane phospholipids by eicosapentaenoic acid (EPA), and the inhibition of cyclooxygenase (EC 1.14.99.1) by EPA. Although one study showed reduced synthesis of PGE and $\mathrm{PGF}_{2 \alpha}$ in tumours of EPA-treated animals, the study failed to show evidence of displacement of AA by EPA in membrane phosphatidylcholine in the EPA-treated groups (Karmali et al. 1984). Measurement of phosphatidylinositol fatty acids may be more relevant, since this membrane component has an important role in hormone signal transduction mechanisms and is suggested to be the major substrate for cell-stimulated release of arachidonic acid in eicosanoid synthesis (Broakman et al. 1980). In normal mammary tissue the fatty acid composition of phosphatidylinositol (PI) appears to be highly conserved, so that unlike the phosphatidylethanolamine (PE) and phosphatidylcholine (PC) fractions, PI fatty acid composition remains constant across a range of fatty acid intakes (Williams \& Maunder, 1992). However, it is not known whether the ability to maintain a constant PI fatty acid composition is retained in tumour tissue. If this homeostatic mechanism is lost in the abnormally dividing mammary cell then the PI pathway could provide a route through which permissive effects of dietary fat on hormone sensitivity could act. In order to investigate this possibility we have compared the susceptibility to dietary modification of mammary PI in tumour- and non-tumour-bearing animals fed on maize, olive and fish oils from the time of tumour induction. Effects of dietary fat composition on PI fatty acids were compared with effects on the major membrane phospholipid, PE. The effect of dietary fatty acid composition on mammary tumour incidence has also been studied.

\section{MATERIALS AND METHODS}

\section{Animals and diet}

Forty-eight, 30-d-old female Sprague-Dawley rats weighing approximately $80 \mathrm{~g}$ were divided into three groups of sixteen animals to study effects of mammary tumourigenesis and dietary fatty acid composition on membrane phospholipids and effects of diet on mammary tumour incidence. Animals received either olive oil, maize oil or fish oil (MaxEPA) diets from the time of tumour induction. Animals were housed in groups of four in a room with controlled temperature, humidity and lighting. Animals had free access to water and the experimental diets. The basal diets comprised $(\mathrm{g} / \mathrm{kg}): \operatorname{starch} 350$, sucrose 300 , casein 200, solka floc 40, mineral mix $40\left(\mathrm{CaHPO}_{4}, \mathrm{Na}_{2} \mathrm{HPO}_{4}, \mathrm{CaCO}_{3}, \mathrm{MgSO}_{4}, \mathrm{ZnCO}_{3}\right.$, $\mathrm{FeSO}_{4} .7 \mathrm{H}_{2} \mathrm{O}, \mathrm{CuSO}_{4} .5 \mathrm{H}_{2} \mathrm{O}, \mathrm{MnSO}_{4} . \mathrm{H}_{2} \mathrm{O}$ ), vitamin mix (nicotinic acid, calcium- $d$ pantothenate, thiamin hydrochloride, riboflavin, pyridoxine, pteroylmonoglutamic acid, $d$ biotin, cyanocobalamin, choline bitartrate) 20 and methionine 2. Oils (maize oil, olive oil 
or MaxEPA) were added to the basal diet to provide $40 \mathrm{~g} / \mathrm{kg}$ diet. The diets were mixed weekly and stored in the dark at $-20^{\circ}$ until required. Food pots were changed daily and used food pots washed thoroughly to remove any adherent dried diet. These procedures were undertaken to ensure maximal stability of the MaxEPA-containing diet in order to prevent lipoperoxidation. Daily food intakes and weekly body weights were monitored for all the animals.

\section{Tumour induction}

Half the animals in each dietary group were randomly allocated to receive the carcinogen $N$-ethyl- $N$-nitrosourea (ENU; SIGMA chemicals, Dorset) by oral administration at a dose of $180 \mathrm{mg} / \mathrm{kg}$.

Animals were tail-marked for identification purposes, numbered 1 to 8 within each treatment (carcinogen or non-carcinogen) and dietary (maize, olive or fish oil) group. Development of mammary tumours was monitored weekly; palpable tumours began to be detected at 9 weeks after carcinogen administration. When a palpable tumour was felt the tumour-bearing animal and a control animal from the same dietary group were killed. The animal killed in the control group was the animal marked with the same number (1 to 8) on entry into the study as the tumour-bearing ENU-treated animal. Mammary tumour and normal tissue was excised and weighed and taken to the laboratory for immediate extraction of total lipids. The experiment was continued for 20 weeks; carcinogen-treated animals which had not developed tumours by this time were killed as above and a full autopsy was performed. One animal from the fish-oil group died at 16 weeks; no mammary tumour or other abnormality was found at autopsy. This animal has been excluded from the analysis, so that in the fish-oil ENU-treated group seven animals completed the experiment according to the protocol.

\section{Laboratory procedures}

Extraction of total lipids from tissue and separation of individual phospholipid classes in the lipid phases prepared from both normal and tumour tissues was achieved by two-stage TLC as previously described (Williams et al. 1989). Bands corresponding to the ethanolamine- and inositol-phospholipids were removed from the plated sample, the phospholipids hydrolysed, fatty acids methylated and subsequently analysed by GLC. Butylated hydroxytoluene (BHT) was added to all extraction solvents at a concentration of $50 \mathrm{mg} / 1$, and to all TLC solvents at a concentration of $500 \mathrm{mg} / 1$ to minimize autooxidation of polyunsaturated fatty acids. Further precautions were taken by storing the lipid extracts in chloroform under $\mathrm{N}_{2}$ at $-20^{\circ}$ and methyl esters in hexane under $\mathrm{N}_{2}$ at $-20^{\circ}$. GLC analyses were carried out using a Varian 3400 instrument, using a $6 \mathrm{~mm} \times 1.5 \mathrm{~m}$ glass column ( $10 \mathrm{~g}$ Silar $10 \mathrm{C} / 100 \mathrm{~g}$ Gas Chrom Q $(100-120 \mathrm{mesh}))$; initial oven temperature $165^{\circ}$, increased to $215^{\circ}$ at $5^{\circ} / \mathrm{min}$; injection temperature $250^{\circ}$; detector $275^{\circ} ; \mathrm{N}_{2}$ flow-rate $30 \mathrm{ml} / \mathrm{min}$.

Results for individual fatty acids are expressed as a percentage of total fatty acids detected $(\mathrm{mol} \%)$.

\section{Statistical analysis of results}

The results in the tables are presented as mean values with their standard deviations. Figures show mean values with their standard errors. Analysis of variance was used to determine whether there were any differences in membrane composition between the three dietary groups or between tumour- and non-tumour-bearing animals. Duncan's range test was used to determine the level of significance of any differences found. $P<0.05$ was considered to be statistically significant. Where data were found to have an unacceptable level of variance using Cochrane's $\mathrm{C}$, Bartlett Box or maximum/minimum variance tests, 
Table 1. Mean food intakes ( $\mathrm{g} / \mathrm{rat}$ per week) and body weights ( $\mathrm{g}$ ) at culling in control and ENU-treated rats fed on olive oil, maize oil and fish oil diets

(Values are means and standard deviations)

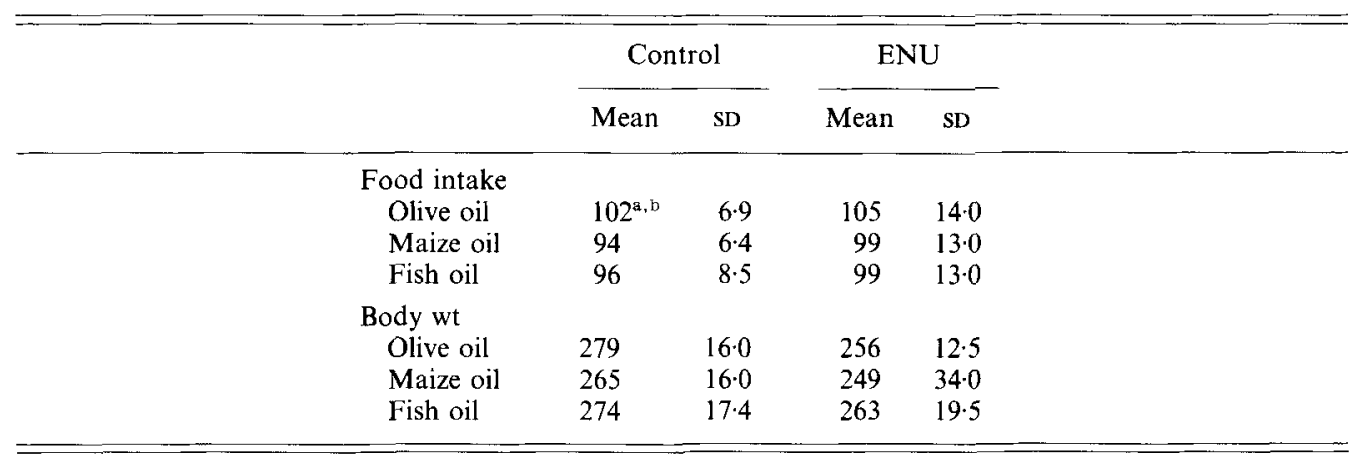

a Value significantly different from maize oil control, $P<0.05$.

b Value significantly different from fish oil control, $P<0.05$.

Table 2. Tumour incidence and tumour latency in ENU-treated animals fed on olive oil, maize oil and fish oil diets

(Mean values and standard deviations)

\begin{tabular}{|c|c|c|c|c|c|c|}
\hline & \multicolumn{2}{|c|}{ Olive oil } & \multicolumn{2}{|c|}{ Maize oil } & \multicolumn{2}{|c|}{ Fish oil } \\
\hline & Mean & SD & Mean & SD & Mean & SD \\
\hline Percentage of tumour-bearing animals & 75 & & 63 & & $29^{\mathrm{a}}$ & \\
\hline $\begin{array}{l}\text { No. of animals with tumours/ } \\
\text { no. of animals treated }\end{array}$ & $6 / 8$ & & $5 / 8$ & & $2 / 7$ & \\
\hline Time to appearance of tumour (d) & 118 & $21 \cdot 4$ & 106 & $2 \cdot 6$ & 137 & $3 \cdot 5$ \\
\hline
\end{tabular}

a Value significantly different from olive oil, $P<0.04$.

analysis was performed on log-transformed or square root-transformed data. Fisher's exact probability test was used to determine whether there were any differences in tumour incidence between fish oil-fed compared with corn oil- or olive oil-fed animals.

Values for stearic and oleic acids and for $n-6$ and $n-3$ fatty acids are presented as bar diagrams. No statistically significant differences in any of the other fatty acids detected, but not presented here, were found between dietary groups or between control or tumourbearing animals.

\section{RESULTS}

Food intake was significantly higher in the olive oil control group than in control animals fed on maize oil or fish oil diets ( $P<0.05$, both cases). In ENU-treated animals fed on olive oil the mean food intake was numerically higher than in the other two groups, but this difference was not statistically significant (Table 1). Cumulative tumour incidence at 20 weeks was significantly lower in the fish oil-fed animals than in the olive oil-fed group ( $P$ $<0.04$; Table 2), but differences between fish oil- and maize oil-fed animals failed to reach a level of statistical significance $(P<0 \cdot 1)$. Tumour incidence rates were $75 \%, 63 \%$ and $29 \%$ in olive oil, maize oil and fish oil groups respectively. Mean tumour latency (Table 2) 

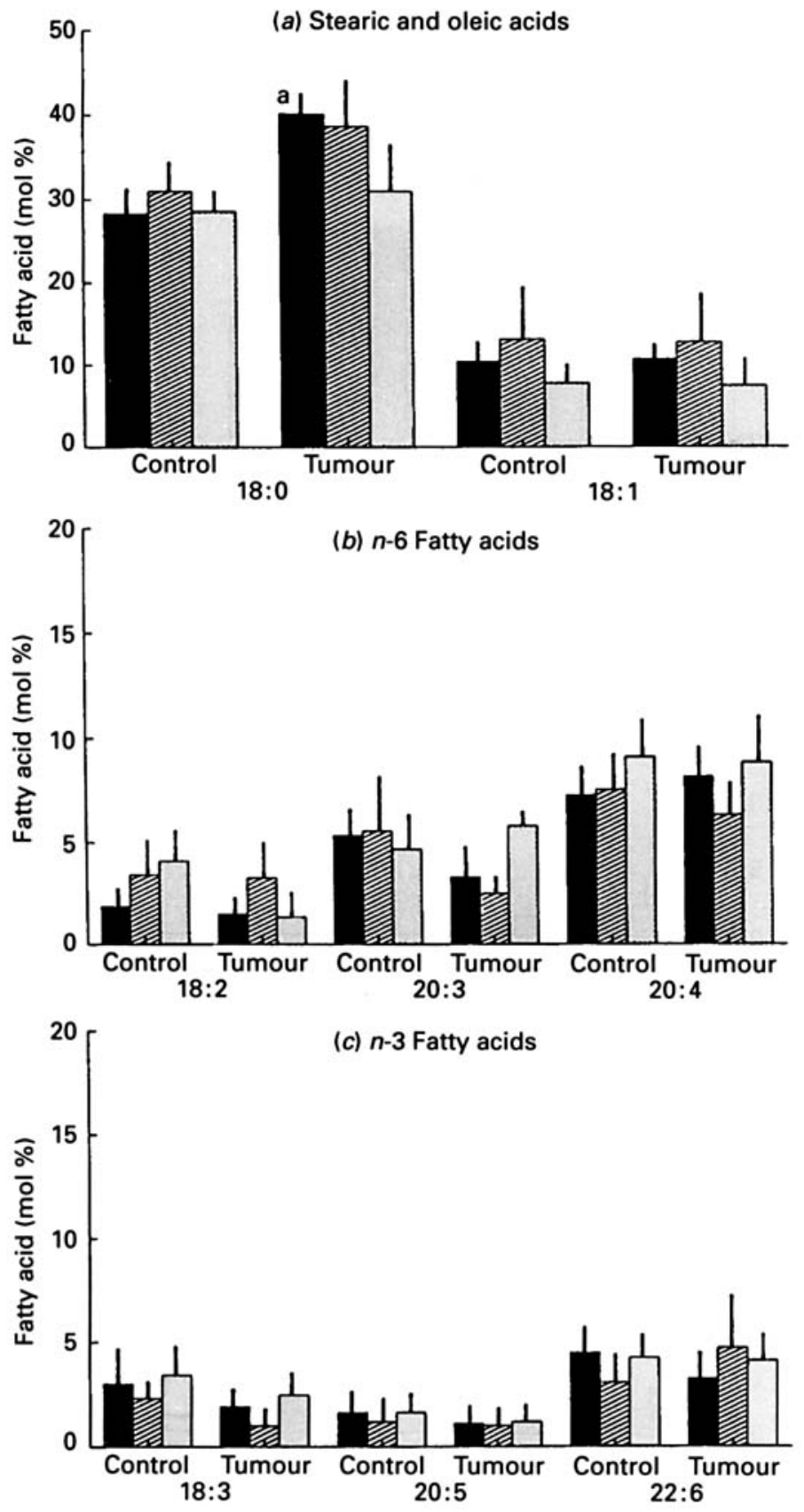

Fig. 1. Mammary phosphatidylinositol fatty acid composition in normal and tumour tissue of animals fed on maize oil (圈), olive oil (n) and fish oil (圈) diets from tumour induction. Values are means with their standard errors; $n 8$ in each control group; $n 6$ olive oil tumour; $n 5$ maize oil tumour; $n 2$ fish oil tumour. ${ }^{2}$ Value significantly different from olive oil control $(P<0.05)$. 

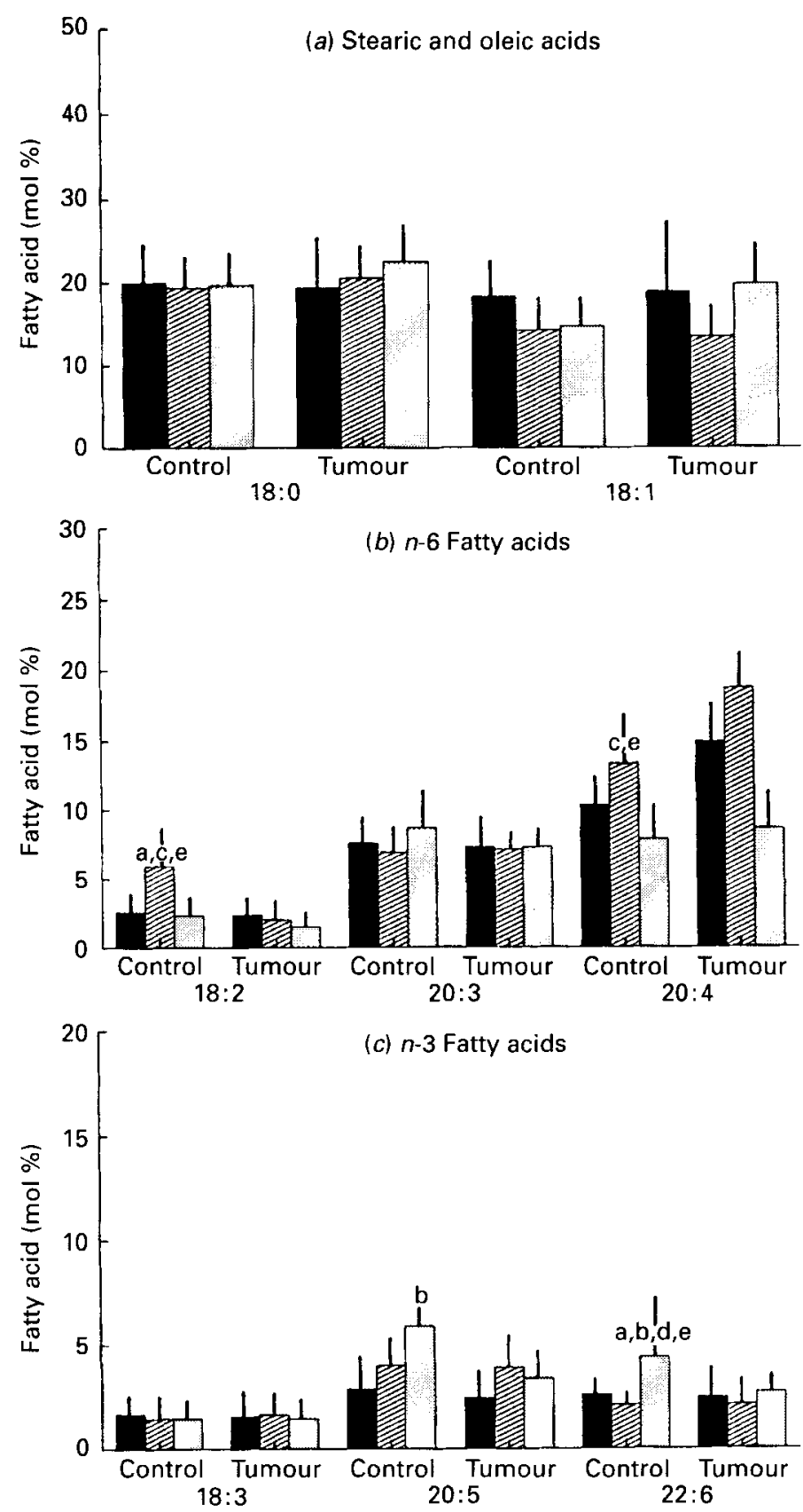

Fig. 2. Mammary phosphatidylethanolamine fatty acid composition in normal and tumour tissue of animals fed on maize oil (国), olive oil ( $\square$ ) and fish oil (圈) diets from tumour induction. Values are means with their standard errors. ${ }^{a}$ Value significantly different from olive oil control $(P<0.05)$, ${ }^{\mathbf{b}}$ value significantly different from maize oil control $(P<0.05)$, "value significantly different from fish oil control $(P<0.05)$, "value significantly different from olive oil tumour $(P<0.05)$, ${ }^{e}$ value significantly different from maize oil tumour $(P<0.05)$. 
was extended in the fish oil group compared with the other two groups, but the small number of animals developing tumours in the fish oil group (two) precluded the use of statistical analysis in comparing these data. The first palpable tumour was found in olive oil-fed animals at 9 weeks, with the majority of tumours appearing between 15 and 20 weeks after induction.

There were no differences in mammary PI fatty acids between the three dietary groups for either normal or tumour tissue (Fig. 1). However, PI stearic acid content was significantly higher in tumours of olive oil-fed animals than that of normal tissue in animals fed on the same diet $(P<0 \cdot 05 ;$ Fig. 1 $a)$. Tumour PI stearic acid content was also numerically higher in tumours than normal tissue of maize oil-fed animals, but this difference failed to reach statistical significance $(P<0 \cdot 1)$.

In the PE fraction of normal tissue from control animals fed on maize oil the levels of linoleic acid were significantly higher than in either olive oil- $(P<0.05)$ or fish oil-fed control animals $(P<0.05)$, and in this group levels of AA were also higher than in fish oilfed control animals $(P<0.05$; Fig. $2 b)$. Fish oil-fed control animals had significantly higher levels of EPA than maize oil controls and of docosahexaenoic acid (DHA) than both maize oil and olive oil control groups $(P<0.05$, all cases; Fig. $2 c)$. In tumour-bearing animals there were no statistically significant differences in PE fatty acid composition between the dietary groups. The mean value for AA was numerically smaller in the fish oil$(8.6 \mathrm{~mol} \%)$ than maize oil- $(18.1 \mathrm{~mol} \%)$ fed animals but this difference did not reach a level of significance due to the small number of animals developing tumours in the fish oil group. In tumour-bearing animals given maize oil, PE linoleic acid content was lower $(P<0.01)$, and AA content was higher $(P<0 \cdot 05)$, than in normal tissue from control animals fed on the same diet.

\section{DISCUSSION}

This study has demonstrated a significantly lower incidence of mammary tumours 20 weeks after carcinogen administration in animals given fish oils as the sole source of dietary fat from the time of tumour induction, compared with animals given olive oil diets. The percentage of tumours at 20 weeks was higher in the maize oil than the fish oil group but the differences did not reach a level of statistical significance. These findings are in agreement with those of previous studies which have shown protective effects of fish oils in carcinogen-induced (Jurkowski \& Cave, 1985) and transplantable (Karmali et al. 1984) mammary tumour models. The time to first appearance of tumours was shorter in the maize oil and olive oil groups than in the fish oil group suggesting, as in previous studies, that feeding fish oils slows down the rate of tumour development but does not completely abolish tumour formation. Had the experiment continued beyond 20 weeks it is possible that all animals in each of the dietary groups would have eventually developed tumours.

A few studies have investigated effects of olive oil on mammary tumourigenesis and have concluded that this oil does not have the same tumour-enhancing effects as oils containing large amounts of $n-6$ fatty acids (Tinsley, 1989). The results obtained in the present study suggest that olive oil has the same capacity as maize oil to support the growth of mammary tumours. However, care should be taken in the interpretation of tumour incidence rates in olive oil-fed animals in the present study, since olive oil-fed control animals were found to have higher food intakes than animals in the other two dietary groups. The mechanism by which dietary fat influences mammary tumourigenesis in experimental animals is not fully understood, but tumour growth is known to be influenced by both the level and type of fat fed, with highest rates of tumourigenesis seen in animals given dietary fat at levels greater than $20 \%$ of total energy (Welsch, 1987). Early animal studies which used isoenergetic, high- and low-fat diets suggested that effects of dietary fat were independent of energy 
(Hopkins \& Carroll, 1979; Cohen et al. 1984). However, Donato \& Hegsted (1985) have proposed that experiments designed to investigate isoenergetic diets should take into account the relatively greater efficiency of utilization of dietary fat, by using a net energy value for fat $(48.5 \mathrm{~kJ})$ rather than the Atwater value $(37.6 \mathrm{~kJ})$. Using this approach Boissonneault et al. (1986) showed mammary tumour incidence to be directly proportional to the net energy content of diets. More recently, Welsch et al. (1990) have shown that differences in tumour incidence in high- and low-fat-fed animals are only observed when animals are allowed to feed freely; moderate energy restriction abolished differences in tumour incidence between high- and low-fat groups.

Consideration of the results from these studies suggests that at least some of the tumourenhancing effects of high-fat diets on mammary tumourigenesis are due to provision of excess calories. Since in the present study food intakes were higher in olive oil-fed control animals, higher rates of tumourigenesis in ENU-treated olive oil-fed animals could be due to higher energy intakes before tumour appearance.

Previous studies suggested that inhibitory effects of fish oil diets on mammary tumour formation are due to the ability of EPA to act as a substrate for the formation of less potent prostaglandins than those produced from AA (Karmali, 1987). The precise mechanism by which prostanoids are involved in abnormal proliferation in mammary tumourigenesis is not understood, but human breast tumours synthesize large amounts of $\mathrm{PGE}_{2}$ and $\mathrm{PGF}_{2 \alpha}$ (Rolland et al. 1979) and, in animal studies, inhibition of prostanoid synthesis with indomethacin has been shown to inhibit effects of high-fat diets on carcinogen-induced mammary tumours (Carter et al. 1983; Kollmorgen et al. 1983). Inhibitory effects of $n-3$ fatty acids and fish oils on mammary tumourigenesis have been attributed to inhibition of cyclo-oxygenase by EPA, and to reductions in membrane AA content through displacement of $\mathrm{AA}$ and inhibition of the $\triangle$-6-desaturase enzyme (EC 1.14.99.6) (which regulates the key step in the conversion of linoleic acid to AA) by EPA. Alteration in membrane AA and EPA content might, therefore, be expected to be seen in tissues in which EPA-induced inhibition of prostanoid metabolism is proposed to occur. Previous authors have shown slight increases in EPA and DHA in total (Jurkowski \& Cave 1985), and choline- (Karmali et al. 1984) phospholipids in mammary tissue of animals given fish oils, but in neither study was a significant alteration in membrane AA content observed.

In many tissues, including mammary tissue, hormone-stimulated PI breakdown is thought to be the most important source of AA for synthesis of series-2 prostanoids. It is for this reason that we have studied the effect of dietary fatty acid modification on the composition of membrane PI as well as on the composition of PE, a more widely investigated phospholipid fraction. Although we have previously shown normal mammary tissue PI to be unresponsive to dietary fat modification (Williams \& Maunder, 1992), it was not known whether this homeostatic ability is lost in tumour tissue. The findings presented here show that this is not the case; in particular there was no evidence of displacement of AA by EPA in fish oil-fed control or tumour-bearing animals, so that it is unlikely that proposed effects of dietary fat on tumourigenesis through altered prostanoid synthesis are the result of diet-induced changes in PI fatty acids. However, results obtained in the present study for effects of both diet and tumourigenesis on PE fatty acid composition are not inconsistent with an involvement of prostanoid metabolism in effects of dietary fat on mammary tumourigenesis and suggest the likely locus for these effects may be PE rather than PI. In maize oil-fed animals levels of linoleic acid were lower and levels of AA were higher in tumour compared with non-tumour tissue. These results suggest that conversion of linoleate to AA may be increased in mammary tumour PE, and that this may be the result of increased $\Delta$-6-desaturase activity in tumour cells. It should be noted, however, that failure to observe diet-induced alteration in PI AA content does not exclude the possibility 
that this phospholipid plays a functionally important part in the tumourigenic process. AA turnover in this fraction may be greater than in PE, so that higher AA content of the latter may reflect lower metabolic activity of PE compared with PI. Labelled AA turnover studies of PI and PE fractions would be required to determine whether this is the case.

In the PE fraction, fish oil-fed control animals had significantly lower levels of AA and higher levels of EPA and DHA than did maize oil-fed animals, and a similar trend was seen in tumour-bearing animals, suggesting that in these animals PE AA was displaced by longchain $n-3$ fatty acids. However, the differences that we have observed are small and our results suggest that fatty acids in mammary tissue phospholipids are less susceptible to dietary modification than has been shown for other tissues such as liver and platelets (Weiner \& Sprecher, 1984; Gibney \& Bolton-Smith, 1988).

Beneficial effects of $n-3$ fatty acids on other tissues such as platelets are generally attributed to the ability of these fatty acids to displace $n-6$ fatty acids from membrane phospholipids, with consequent effects on prostanoid metabolism. However, a number of studies show that $n-3$ fatty acid supplementation causes alteration in membrane fatty acids other than the $n-6$ fatty acids. For example, in platelets Gibney \& Bolton-Smith (1988) showed a significant change in platelet PC oleic acid content in subjects taking fish oil supplements, and we have also observed marked differences in erythrocyte saturated fatty acid composition in rats fed on fish oils (Williams \& Maunder, 1992), suggesting that $n-3$ fatty acid supplementation produces effects other than simple displacement. The possible functional consequences of these effects require further consideration.

An unexpected observation in the present study was that in olive oil-fed animals PI stearic acid content was significantly higher in tumour than normal tissue, and a similar trend was apparent in tumour tissue of maize oil-fed animals. No such effect was observed in fish oil-fed animals. This observation may be important since we have also found markedly higher PI stearic acid levels in malignant human breast tissue compared with levels in benign tumours or normal breast tissue (Williams, 1992). It is possible that altered stearic acid content of membrane PI may influence the sensitivity of the PI signal transduction mechanism through alteration in its binding to the $G$ unit coupling protein which brings about receptor-mediated activation of PI hydrolysis. It is also possible that PI stearic acid content may influence the potency of the diacylglycerol released following PI breakdown, since the acyl chain composition of this second messenger has been suggested to influence its activity (Welsch, 1987).

In summary, results obtained in the present study suggest that mammary tumour tissue phospholipids respond to diet in a similar manner to normal mammary tissue. Mammary membrane PI composition is not altered in response to diet, but mammary membrane PE AA is susceptible to displacement with long chain $n$-3 fatty acids. Mammary tumour tissue fatty acid composition was found to be similar to normal mammary tissue, although there was evidence of increased stearic acid content of PI in olive oil-fed animals. Further studies of the stearic acid content of tumour tissue PI and possible consequences on the sensitivity of the PI signal transduction mechanism would appear to be warranted.

We are grateful to Dr R. J. Howland for advice and assistance with statistical analysis.

\section{REFERENCES}

Berrino, F. \& Muti, P. (1989). Mediterranean diet and cancer. European Journal of Clinical Nutrition 43, 49-55. Boissonneault, G. A., Elson, C. E. \& Pariza, M. W. (1986). Net energy effects of dietary fat on chemically induced mammary carcinogenesis in F344 rats. Journal of the National Cancer Institute 76, 335-338.

Broakman, M. J., Ward, J. W. \& Marcus, A. J. (1980). Phospholipid metabolism in stimulated human platelets. Changes in phosphatidylinositol, phosphatidic acid and lysophospholipids. Journal of Clinical Investigation 66, 275-283. 
Carroll, K. K. (1975). Experimental evidence of dietary factors and hormone-dependent cancers. Cancer Research 35, $3774-3783$.

Carter, C. A., Milholland, R. J., Shea, W. \& Ip, M. M. (1983). Effect of prostaglandin synthetase inhibitor indomethacin on 7, 12 dimethylbenz (a) anthracene-induced mammary tumorigenesis in rats fed different levels of fat. Cancer Research 43, 3559-3562.

Cohen, L. A., Thompson, D. O., Maeura, Y. \& Weisburger, J. H. (1984). Influence of dietary medium chain triglycerides on the development of $N$-methylnitrosourea-induced rat mammary tumors. Cancer Research 44 , 5023-5028.

Donato, K.\& Hegsted, D. M. (1985). Efficiency of utilisation of various sources of energy for growth. Proceedings of the National Academy of Sciences, USA 82, 4866-4870.

Gibney, M. J. \& Bolton-Smith, C. (1988). The effect of a dietary supplement of $n-3$ polyunsaturated fat on platelet lipid composition, platelet function and platelet plasma membrane fluidity in healthy volunteers. British Journal of Nutrition 60, 5-12.

Hopkins, G. J. \& Carroll, K. K. (1979). Relationship between the amount and type of dietary fat in promotion of mammary carcinogenesis induced by 7,12 , dimethylbenz (a) anthracene. Journal of the National Cancer Institute 62, 1009-1012.

Jurkowski, J. J. \& Cave, W. T. (1985). Dietary effects of Menhaden oil on the growth and membrane lipid composition of rat mammary tumours. Journal of the National Cancer Institute 74, 1145-1150.

Karmali, R. A. (1987). Fatty acids: inhibition. American Journal of Clinical Nutrition 45, 225-229.

Karmali, R. A., Marsh, J. \& Fuchs, C. (1984). Effects of $\omega-3$ fatty acids on the growth of a rat mammary tumour. Journal of the National Cancer Institute 73, 457-461.

Kollmorgen, G. M., King, M. M., Kosanke, D. \& Do, C. (1983). Influence of dietary fat and indomethacin on the growth of transplantable mammary tumors in rats. Cancer Research $\mathbf{4 3 , 4 7 1 4 - 4 7 1 9 .}$

Kritchevsky, D., Weber, M. M. \& Klurfeld, D. M. (1984). Dietary fat versus calorie content in initiation and promotion of 7, 12-dimethylbenz (a) anthracene in mammary tumorigenesis in rats. Cancer Research 44, $3174-3177$.

Lea, A. J. (1966). Dietary factors associated with death-rates from certain neoplasms in man. Lancet ii, 332-333.

McMichael, A. J. \& Potter, J. D. (1985). Diet and colon cancer: integration of the descriptive, analytic and metabolic epidemiology. National Cancer Institute Monographs 69, 223-228.

Potter, J. D. (1987). Dietary fat and the risk of breast cancer. New England Journal of Medicine 317, 166.

Prentice, R. L., Kakar, F., Hursting, S., Sheppard, L., Klein, R. \& Kushi, L. H. (1988). Aspects of the rationale for the women's health trial. Journal of the National Cancer Institute 80, 802-814.

Rolland, P. H., Martin, P. M., Rolland, A. M., Bourry, M. \& Serment, H. (1979). Benign breast disease: studies of prostaglandin $E_{2}$, steroids and the thermographic effects of inhibitors of prostaglandin biosynthesis. Obstetrics and Gynecology 54, 715-719.

Tinsley, I. J. (1989). Dietary fatty acids and mammary tumorigenesis. In: Carcinogenesis and Dietary Fat, pp. 101-113 [S. Abraham, editor]. Boston: Kluwer Academic Publishers.

Weiner, T.W. \& Sprecher, H. (1984). Arachidonic acid, 5,8,11-eicosatrienoic acid and 5,8,11,14,17eicosapentaenoic acid. Dietary manipulation of levels of these acids in rat liver and platelet phospholipids and their incorporation into human platelet lipids. Biochimica et Biophysica Acta 792, 293-303.

Welsch, C. W. (1987). Enhancement of mammary tumorigenesis by dietary fat: review of potential mechanisms. American Journal of Clinical Nutrition 45, 192-202.

Welsch, C. W., House, J. L., Herr, B. L., Eliasberg, S. J. \& Welsch, M. A. (1990) Enhancement of mammary carcinogenesis by high levels of dietary fat: A phenomenon dependent upon ad-libitum feeding. Journal of the National Cancer Institute 82, 1615-1620.

Williams, C. M. (1992). Diet, lipids and breast cancer. In Food, Nutrition and Chemical Toxicity, pp. 353-361 [D. V. Parke, C. Ionnides and R. Walker, editors]. London: Smith-Gordon.

Williams, C. M. \& Maunder, K. (1992). Effect of dietary fatty acid composition on inositol-, choline- and ethanolamine-phospholipids of mammary tissue and erythrocytes in the rat. British Journal of Nutrition $\mathbf{6 8}$, $183-193$

Williams, C. M., Maunder, K. \& Theale, D. (1989). The effect of a low-fat diet on luteal-phase prolactin and oestradiol concentrations and erythrocyte phospholipids in normal premenopausal women. British Journal of Nutrition 61, 651-661. 